\title{
Estresse térmico e o desempenho produtivo de ovinos: uma revisão
}

[Thermal stress and the productive performance of sheep: a review]

\section{"Revisão/Review"}

\author{
Volmir Antonio Polli ${ }^{1}$, Pablo Tavares Costa ${ }^{2 *}$, João Restle ${ }^{3}$, Rafael Bonadiman ${ }^{4}$, \\ Ricardo Zambarda $\mathbf{V a z}$
}

${ }^{1}$ Colégio Politécnico de Santa Maria, Santa Maria-RS, Brasil.

${ }^{2}$ Departamento de Zootecnia, Universidade Federal de Pelotas, Pelotas-RS, Brasil.

${ }^{3}$ Escola de Veterinária e Zootecnia, Universidade Federal de Goiás, Goiânia-GO, Brasil.

${ }^{4}$ Instituto Federal Farroupilha, Santa Maria-RS, Brasil.

*Autor para correspondência/Corresponding author: E-mail: pablocostta@ hotmail.com

\section{Resumo}

O clima interfere de maneira marcante na produtividade animal, característica que tem sua importância ressaltada no tocante às recentes mudanças climáticas verificadas e às previsões de aumento no aquecimento global. Neste contexto, a presente revisão visa abordar os efeitos do clima sobre o conforto térmico, aspectos comportamentais e desempenho produtivo em ovinos. As condições climáticas acarretam respostas fisiológicas e comportamentais relevantes nos ovinos, e, quando essas condições atingem graus elevados de estresse, acabam impactando fortemente nos índices de produtividade. É necessário adequar práticas de manejo, instalações e alimentação de tal forma que permitam melhores condições de bem-estar dos animais frente às condições climáticas estressantes, bem como selecionar biótipos mais adaptados às características climáticas, as quais serão explorados, buscando permitir que expressem todo seu potencial produtivo.

Palavras-chave: fatores climáticos; bem-estar; ovinocultura.

\begin{abstract}
Climate has a marked effect on animal productivity, a feature that has its importance highlighted in relation to the recent verified climate changes and the predictions of increase in global warming. In this context, the present review aims to discuss the effects of climate on the thermal comfort, behavioral aspects and productive performance of sheep. Climatic conditions result in relevant physiological and behavioral responses in sheep, and when these conditions reach high levels of stress, they have a strong impact on productivity indices. It is necessary to adapt management practices, installations, and feeding to allow better animal welfare conditions in the presence of stressful climatic conditions, as well as to select biotypes that are more adapted to the climatic characteristics of the productive system, in order to allow expression of the animals' full productive potential.
\end{abstract}

Keywords: climatic factors; welfare; sheep farming.

\section{Introdução}

Durante várias décadas a busca pela máxima eficiência na produção animal foi focada em necessidades relacionadas ao manejo, sanidade, genética e nutrição (Bridi, 2010a). Contudo, atualmente, os avanços conquistados nestas características têm sido limitados pelos fatores ambientais, principalmente pelo ambiente térmico

aos quais os animais, estão submetidos (Kadzere et al., 2002; Marai et al., 2007; Bridi, 2010a; Rodrigues et al., 2010). Assim, um dos grandes desafios da pecuária moderna está relacionado à exploração do máximo potencial genético dos animais que necessita de condições adequadas de conforto térmico para ser expresso. 
Ovinos são animais endotérmicos, ou seja, mantêm a temperatura corporal dentro de certos limites fisiológicos relativamente estreitos pelo controle da produção e perda de calor (Eckert, 2000), mesmo que sua atividade varie e que a temperatura ambiente flutue (Baêta e Souza, 2012). Ovinos são considerados em conforto térmico quando seu índice de temperatura e umidade (ITU) é inferior a 22,2 (Marai et al., 2007). Fora desta zona de conforto, sua fisiologia e comportamento sofrem alterações, com consequências negativas de desempenho (Eckert, 2000; Baumgard e Rhoads, 2012). O conhecimento das diferentes respostas fisiológicas e comportamentais que o animal apresenta quando em estresse calórico, bem como suas consequências em termos produtivos, são importantes no intuito de avaliar e quantificar sua adaptabilidade ao ambiente.

A influência dos fatores climáticos nos aspectos produtivos dos animais adquire maior importância no tocante às mudanças climáticas verificadas recentemente ao redor do planeta, bem como às previsões de aumento no aquecimento global (Baumgard e Rhoads, 2012). Essa revisão visa abordar os efeitos do clima no conforto térmico, em aspectos comportamentais e no desempenho produtivo de ovinos.

\section{O clima e a produção de ovinos}

Os animais portam-se como um sistema termodinâmico, que continuamente troca energia com o ambiente. Neste processo, os fatores externos do ambiente tendem a produzir variações internas no animal, influindo na quantidade de energia trocada em ambos, havendo então a necessidade de ajustes fisiológicos para a ocorrência de balanço térmico (Baêta e Souza, 2012).

Ovinos possuem altas taxas metabólicas e ainda assim precisam ser capazes de manter a temperatura corporal acima da temperatura ambiente, regulando, dentro de limites fisiológicos estritos, pelo controle da produção e perda de calor (Eckert, 2000; Moyes e Shulte, 2010; Baêta e Souza, 2012). Quanto maior a eficiência do animal no uso das fontes de energia disponíveis no ambiente, maior a capacidade de competir com outros membros de sua espécie, no sentido evolucionário (Eckert, 2000). Dessa forma, a adaptabilidade dos animais de criação frente às condições do meio ambiente é uma das características importantes quando se busca eficiência produtiva. Assim, a espécie ovina acaba tendo uma posição de destaque, pois é tida como uma das mais adaptadas entre as diversas espécies domésticas criadas pelo homem (Marai et al., 2007).

O tamanho corporal dos ovinos, considerado pequeno, dá aos mesmos, relação favorável entre a área da superfície e o volume para a perda de calor (Moyes e Schulte, 2010). Os ovinos lanados podem ser considerados animais com boa adaptação aos mais distintos climas, devido a sua excelente capacidade de isolamento térmico (Degen e Shkolnik, 1978). No entanto, apesar da versatilidade de adaptação aos diferentes meios, ovinos também sofrem estresse calórico, notadamente quando elevadas temperatura e umidade relativa do ar estão associadas (Starling et al., 2002).

Muitos estudos científicos têm mostrado que o aquecimento global é uma realidade, especialmente no que tange as flutuações nos padrões climáticos, particularmente nas chamadas ondas de calor que são hoje mais frequentes e severas (Kuczinski et al., 2011). O aumento na temperatura em escala global tem efeito direto nos animais, representado pelo estresse calórico, o qual tem uma variedade de efeitos negativos sobre a fisiologia, saúde, bem-estar e produtividade dos mesmos (Marai et al., 2007; Kuczinski et al., 2011; Papanastasiou et al., 2015), podendo assim, reduzir os lucros do produtor de maneiras distintas (Papanastasiou et al., 2015). Nas condições de alta temperatura e umidade do ar, os animais têm enormes dificuldades de perda de calor corporal, ou seja, de fazer a termorregulação.

Os efeitos perversos no desempenho, nas distintas fases de vida, serão tão maiores quanto maior e mais duradouro for o estresse climático (St. Pierre et al., 2003). Essas perdas advêm da redução do consumo alimentar, que acarreta menores taxas de crescimento, produção de leite, perdas reprodutivas e aumento da taxa de mortalidade (Kadzere et al., 2002).

A interação animal versus ambiente deve ser considerada quando se busca eficiência na exploração pecuária, pois as diferentes respostas do animal às peculiaridades regionais são determinantes no sucesso da atividade produtiva. Assim, a correta identificação dos fatores que influenciam na vida produtiva do animal, como o estresse imposto pelas flutuações estacionais do meio ambiente, permitem ajustes nas práticas de manejo dos sistemas de produção, possibilitando dar-lhes sustentabilidade e viabilidade econômica (Neiva et al., 2004). 
Em exemplo, ao estudarem cordeiros Texel confinados em duas condições climáticas (calor mais alto e mais baixo) na região Sul do Brasil, Polli et al. (2019) observaram que cordeiros terminados nas condições de calor mais alto e mais baixo experimentaram, respectivamente, $27,7 \mathrm{e}$ $6,5 \%$ do período total de confinamento sob estresse térmico. Resultando em maiores frequências respiratórias e temperaturas na região ocular nos animais terminados em temperaturas mais altas, e maior eficiência no uso da alimentação para os terminados em temperatura mais baixas. Os autores concluíram que as condições climáticas afetaram o desempenho e o comportamento dos cordeiros como resultado do desconforto térmico.

Os elementos climáticos temperatura e umidade do ar, pressão atmosférica e radiação solar são os principais fenômenos que compõem o clima. A velocidade do vento, por sua vez, tem importante papel no conforto térmico, por atuar promovendo a perda de calor do animal pela via convectiva (Blanes et al., 2008). Estes elementos, quando em condições extremas, atuam de forma direta ou indireta sobre os animais de criação tirando-os de sua zona de conforto térmico, assim deprimindo a eficiência de sua produção. Apesar de haver um grande número de fatores como experiência anterior, genética, idade, sexo ou condição fisiológica capazes de modelar a natureza da resposta biológica de um animal a um determinado agente estressor (Hafez, 1973; Silva, 2000; Rodrigues et al., 2010), como pode ocorrer com o estresse calórico.

\section{Bem-estar animal}

É cada vez mais reconhecido o fato de que elevados padrões de bem-estar têm impacto direto e indireto nos custos, na segurança e na qualidade dos produtos de origem animal (Costa, 2000; Oliveira et al., 2008). As práticas de produção pecuária necessariamente devem estar pautadas nos princípios éticos e morais de respeito e de bemestar dos animais (Chulayo e Muchenje, 2015). Sendo consenso de que o bem-estar animal é condição preponderante na melhoria da eficiência dos sistemas de criação (St. Pierre et al., 2003; Eloy, 2007; Rodrigues et al., 2010) e na obtenção de produtos de melhor qualidade (Costa, 2000; Oliveira et al., 2008).

A condição de bem-estar no processo de criação poderá ser fortemente ameaçada por inúmeros fatores geradores de estresse, dentre os quais temos as condições climáticas as quais os animais são submetidos durante as diferentes etapas da criação (St. Pierre et al., 2003; Marai et al., 2007). Em algumas etapas ou em todo o processo de criação, muitos animais são mantidos em ambientes inadequados e/ou em condições restritas de espaço, não permitindo aos mesmos executar os ajustes comportamentais necessários à manutenção da homeostase térmica, devendo estas práticas de produção serem revisadas (Bridi, 2010b). Segundo Silanikove (2000), a condição de bem-estar pode variar desde condição pobre até muito boa, podendo ser definida por medidas discretas, como alterações no nível hormonal, temperatura corporal e comportamento.

Os animais, para vivenciarem um adequado bem-estar, e assim poderem expressar todo seu potencial produtivo, dependem, entre outros fatores, do atendimento a uma faixa de temperatura adequada determinando o conforto térmico (liberdade ambiental). Nesta faixa de temperatura, o animal não precisa produzir ou perder calor corporal e a atividade metabólica é mínima, podendo os mesmos expressarem seu máximo potencial genético (Eloy, 2007; Bridi, 2010a). A zona de conforto térmico é dependente de diversos fatores, sendo alguns ligados ao animal, como peso, idade, estado fisiológico, tamanho do grupo, nível de alimentação e genética e outros ligados ao ambiente como a temperatura do ar, velocidade do vento e umidade do ar (Bridi, 2010a).

\section{Estresse térmico e as respostas fisiológicas consequentes}

O estresse térmico consiste na força exercida pelos componentes do ambiente térmico sobre o organismo, causando nele uma reação fisiológica proporcional à intensidade da força aplicada e à capacidade do organismo em compensar os desvios causados pela mesma (Silva, 2000). O organismo animal apresenta inúmeras respostas fisiológicas que são comumente usadas e aceitas para quantificar o grau de conforto térmico ao qual o animal está submetido (Silanikove, 2000; Marai et al., 2007). Quando submetido a um ambiente estressante, o animal pode ter seu desempenho produtivo e reprodutivo afetado de forma significativa, bem como o sistema imunológico, dependendo da intensidade e persistência do agente estressor (St. Pierre et al., 2003; Eloy, 2007; Rodrigues et al., 2010).

O primeiro sinal de animais submetidos ao estresse calórico é o aumento da frequência respiratória. Este mecanismo fisiológico promove 
a perda de calor por meio evaporativo, sendo o principal mecanismo de termólise em ovinos lanados (Silva, 2000; Starling et al., 2002). Na persistência desta situação de estresse, os mecanismos de termorregulação intensificam-se, e o animal busca reduzir o metabolismo por meio da diminuição da atividade da tireoidiana, produzindo menos tiroxina (Eloy, 2007).

A homeostase é alcançada por coordenação de um conjunto complexo de processos fisiológicos por meio de comunicação química e/ou elétrica entre os tecidos que apresentam respostas apropriadas, tendo os hormônios papel fundamental neste processo (Eckert, 2000).

A energia térmica influencia as interações químicas que afetam a estrutura das macromoléculas e as reações bioquímicas. Consequentemente, a temperatura possui importante efeito sobre todos os processos fisiológicos. Como resultado dos efeitos da temperatura, cada animal apresenta uma estratégia térmica: as combinações de respostas comportamentais, bioquímicas e fisiológicas que garantem que a temperatura corporal se mantenha dentro de um limite aceitável (Moyes e Schulte, 2010; Souza e Batista, 2012).

A termorregulação apresenta-se como um mecanismo fundamental para a adaptação e manutenção de espécies animais em diferentes habitats, sendo ajustada por dois mecanismos que atuam em conjunto, o sistema endócrino e o sistema nervoso. Ambos sistemas enviam mensagens através de fibras aferentes ao centro regulador, o hipotálamo, que processa as informações e envia respostas através de fibras eferentes e neurônios de associação até os órgãos efetores, que produzem os efeitos necessários à regulação da homeostase (Souza e Batista, 2012).

De forma mais detalhada, sabe-se que a fisiologia do estresse tem sua base representada pelo eixo Hipotálamo-Pituitária-Adrenal. Assim, em condições de estresse térmico, além de sua própria temperatura gerada pelos neurônios termossensíveis, o hipotálamo recebe impulsos neurais de transmissores de outras partes do corpo via Sistema Nervoso Central. As células neurossecretoras, ativadas por estímulos neurais, em resposta a uma variedade de estímulos estressantes ao organismo, induzem a produção do hormônio liberador das corticotropinas (CRH) dentro da veia porta. Após serem liberadas para a pituitária anterior, as $\mathrm{CRH}$ estimulam a secreção do hormônio adrenocorticotrópico (ACTH), o qual, por sua vez, provoca a liberação dos hormônios adrenocorticais pelas adrenais (Encarnação, 1992; Eckert, 2000). Dos hormônios adrenocorticais, os glicocorticoides têm forte relação com a síndrome do estresse, exercem função catabólica, inibindo a multiplicação celular de aminoácidos e glicose, bloqueando a síntese de proteínas, diminuindo a taxa de metabolismo (Eloy, 2007) e reduzindo a ingestão de alimentos (Marai et al., 2007). Os glicocorticoides, representados pelo seu principal hormônio, o cortisol, são responsáveis por regular a intensidade da resposta ao estresse e assim na restauração da homeostase (Randall, 2010).

Com a ativação do eixo hipotálamo-adenohipófise-cortex adrenal ocorrem mudanças em quase todo o sistema endócrino. Assim, o organismo estressado secreta menos hormônio do crescimento (STH), gonadotróficos (FSH) e tireotrófico (TSH) (Encarnação, 1992). Com o aumento da produção de glicocorticoides produzidos pelos rins, o hipotálamo através do TSH deprime sua ação sobre a tireoide e essa para de produzir os hormônios T3 (triiodotironina) e T4 (tiroxina). Estes hormônios são responsáveis pela aceleração do metabolismo e, por conseguinte pelo aumento na produção de calor corporal (Eckert, 2000). Como os glicocorticoides, a secreção dos hormônios tiroidianos (T3 e T4) também é regulada por feedback negativo destes sobre o hipotálamo.

Em situações de estresse, os opioides endógenos como a $\beta$-endorfina e encefalina produzidas pelo sistema nervoso central, também têm importância no controle do apetite e da termorregulação (Eloy, 2007). As catecolaminas (adrenalina e noradrenalina) produzidas pelo córtex adrenal, são responsáveis por dar ao organismo respostas imediatas às situações de estresse (Encarnação, 1992). Em geral, diante de um agente estressor todo o sistema endócrino está envolvido na resposta ao estresse (Rodrigues et al., 2010), agindo de forma integrada no organismo (Eckert, 2000).

Existem ainda várias outras funções fisiológicas desencadeadas pelo sistema neuroendócrino, pelas quais o organismo tenta compensar os efeitos climáticos e manter a homeostasia (Encarnação, 1992). Para a ocorrência destas reações termorreguladoras é desviada energia, que poderia ser empregada para crescimento e reprodução. As principais respostas fisiológicas de adaptação do animal ao calor são a vasodilatação periférica, maior produção de suor, aumento da frequência respiratória, da temperatura da pele e dos batimentos cardíacos e maior ingestão de água e procura por sombra (Encarnação, 1992; Rodrigues et al., 2010). Por outro lado, ocorrem 
diminuições da produção de calor, conversão alimentar, movimentação e consumo de forragem. No entanto, diante de um agente estressor, fatores como genética, idade, sexo ou condição fisiológica acabam ainda modelando a natureza das respostas fisiológicas (Rodrigues et al., 2010).

Pesquisadores são unânimes ao reconhecer a frequência respiratória como a resposta fisiológica do animal que primeiro responde aos efeitos do estresse calórico, sendo a mais correlacionada com o conforto térmico (Kuczinski et al., 2011). Silanikove (2000) mostrou poder quantificar pela avaliação da taxa respiratória a severidade do estresse causado pelo calor em ruminantes. Frequências respiratórias de 40 a 60; 60 a 80 e 80 a 120 movimentos/minuto caracterizam respectivamente, estresse baixo, médio-alto e alto, e em valores acima de 200 movimentos/minuto, o estresse é considerado severo em ovinos.

A temperatura superficial do animal também pode ser usada para estimar o nível de desconforto térmico dos mesmos. Muitas formas de avaliação são usadas, entre elas a termografia de infravermelho e a temperatura do globo ocular (Roberto e Souza, 2014). De maneira geral, as temperaturas obtidas pelo termógrafo infravermelho são bons indicadores das condições de conforto térmico. Porém, essas apresentam grande variação em termos da caracterização do ambiente térmico (Paim et al., 2012), oscilando em função da parte do corpo do animal onde são obtidas e da presença de pelos ou lã, bem como a altura e densidade destes. De acordo com Paim et al. (2012), os pontos preferenciais para obter a situação térmica do animal, que determinaram a maioria dos índices de conforto, são o nariz, o pescoço e a garupa.

Bhattacharya e Hussain (1974) trabalhando com borregas da raça Awasi, mantidas em câmaras climáticas sob diferentes condições de clima e recebendo dietas distintas, verificaram aumento na taxa de respiração (94 x 38 movimentos/minuto), frequência cardíaca $(97$ x 88 bat./min) e temperatura retal $\left(39,1 \times 39,1^{\circ} \mathrm{C}\right)$, respectivamente, nos ensaios com alta temperatura-umidade e ração com $25 \%$ de forragem, e em condições neutras de clima. Quando nas mesmas condições de clima foi incluído na ração $75 \%$ de forragem, os valores foram: $101 \times 43$ movimentos/minuto para frequência respiratória, $110 \times 97$ bat./min para frequência cardíaca e $39,5 \times 39,2^{\circ} \mathrm{C}$ para temperatura retal.

Dixon et al. (1999) ao manterem borregos (Merino X Leicester) em câmaras climáticas durante 44 dias sob duas condições climáticas: temperatura entre 13 a $15^{\circ} \mathrm{C}$ e umidade relativa de 56 a $58 \%$ (frio) e temperatura de 32 a $40^{\circ} \mathrm{C}$ e umidade relativa de 50 a $70 \%$ (calor). Verificaram que a elevação da temperatura teve reflexos na frequência respiratória e temperatura retal $(\mathrm{P}<0,01)$, obtendo, respectivamente, 196 e 56 $\mathrm{resp} / \mathrm{min}$, e 40,1 e $39,2^{\circ} \mathrm{C}$. Srikandakumar et al. (2003) avaliaram a frequência respiratória de borregos Merinos e Omami em condições de estresse térmico em Oman. Os ovinos Merinos apresentaram frequência respiratória de 128 movimentos/min e os Omami 65 movimentos/min. Esta diferença de resposta fisiológica se deve ao grau de adaptação dos animais da raça Omami diante de condições adversas de temperatura ambiente. Em ovinos, a temperatura retal começa a elevar-se acima do normal quando a temperatura ambiente atinge $32^{\circ} \mathrm{C}$ e a ofegação (caracterizada pelo animal apresentar a boca aberta) inicia quando a temperatura atinge $40^{\circ} \mathrm{C}$, estando a umidade relativa do ar abaixo de $65 \%$ (Srikandakumar et al., 2003).

Wojtas et al. (2013) estudaram, na Polônia, ovinos Polish Merino com idade de 12 meses e peso médio de $45 \mathrm{~kg}$, mantidos em três distintos estágios climatológicos de 14 dias cada: em um primeiro momento de termoneutralidade; num segundo período com temperaturas de 30,6 e $28,6^{\circ} \mathrm{C}$ e umidade relativa de 50,5 e $47,8 \%$ durante o dia e a noite, respectivamente; e no terceiro período com idêntica temperatura e umidade relativa ao segundo período, porém com presença de vento a uma velocidade de $3,12 \mathrm{~m} / \mathrm{s}$. Observando diferenças $(\mathrm{P}<0,05)$ quanto a frequência cardíaca $(90,23 ; 107,79$ e 80,8 bat./min), frequência respiratória $(56,21 ; 96,43 \mathrm{e}$ $57,6 \mathrm{mov} . / \mathrm{min})$, temperatura retal $\left(39,36^{\circ} ; 39,36^{\circ} \mathrm{e}\right.$ $\left.38,97^{\circ} \mathrm{C}\right)$ e nível de cortisol sanguíneo $(5,88 ; 7,97$ e $4,48 \mathrm{ng} / \mathrm{dL}$ ), respectivamente, para o primeiro, segundo e terceiro períodos. Resultados estes que salientam a importância do vento, mesmo que em baixas velocidades, como agente redutor do estresse causado por elevadas temperaturas e umidade do ar.

Alhidary et al. (2013) ao trabalharem com borregas da raça Merino com peso médio de 30,4 $\mathrm{kg}$, expostas a altas temperaturas ambientais, verificaram resultados semelhantes aos observados por Wojtas et al. (2013) quanto às variáveis fisiológicas. Estes resultados se associaram a menores consumos diários de matéria seca $(-22 \%)$ e maior consumo de água, passando de 1,3 para 4,4 
(L/d) para as borregas mantidas em condições de alta temperatura.

Os animais endotérmicos utilizam mecanismos básicos para produzir ou perder calor, tais como alterações fisiológicas, comportamentais e metabólicas, com intuito de manter a temperatura corporal relativamente constante (Bridi, 2010b). Estes mecanismos podem ser divididos em duas categorias: os não-evaporativos ou sensíveis e os evaporativos ou latentes (Eckert, 2000). Os meios não-evaporativos incluem a condução, convecção e radiação como formas de trocas de calor, requerendo para sua eficiência um diferencial de temperatura entre o animal e o meio ambiente (Schmidt-Nielsen, 2005). Nos mecanismos evaporativos ou latentes a perda de calor se dá por evaporação da água via respiração ou sudação (Baêta e Souza, 2012). A perda de calor por essa via é o mecanismo mais eficaz por não depender do diferencial de temperatura entre o organismo e a atmosfera (Starling et al., 2002). Já SchmidtNielsen (2005), consideram a ofegação o mecanismo mais eficiente na perda de calor corporal por promover seu próprio fluxo de ar para as superfícies úmidas, facilitando a evaporação. A perda de calor latente através das glândulas sudoríparas constitui-se também em um ótimo mecanismo de adaptação dos ovinos, não tão bom em animais lanados, pois de certa forma pode comprometer a eficiência do mecanismo de sudação por dificultar a evaporação da umidade cutânea (Marai et al., 2007).

\section{Comportamento do ovino diante do estresse}

Juntamente com as respostas fisiológicas, o comportamento que o animal apresenta pode ser considerado um ótimo indicador do grau de conforto térmico em que o mesmo se encontra (Broom e Molento, 2004). Modificações no comportamento são os primeiros sinais visíveis desencadeados por alterações hormonais em decorrência do estresse calórico (Silva et al., 2016). Nessas condições de desconforto é comum os animais manifestarem comportamentos anômalos, os quais se afastam do comportamento natural.

As mudanças comportamentais são primeiramente uma tentativa para manter aceitáveis os níveis de desconforto (Gaughan et al., 2002). Os animais respondem às condições ambientais desfavoráveis de diversas maneiras, destacando-se o movimento ou orientação. Assim, têm possibilidade de alterar seu microclima, buscando encontrar conforto (Baêta e Souza, 2012).

O comportamento ingestivo expresso pelas atividades de alimentação, ruminação, ócio e ingestão de água, e ainda pela posição corporal (em pé ou deitado) durante o dia sofre interferência das condições climáticas (Hafez, 1973). Em condições de severo estresse térmico, com intuito de reduzir a produção de calor corporal, naturalmente os animais reduzem a ingestão de alimento, seu metabolismo e em consequência a produção de calor (Marai et al., 2007). Portanto, a redução na ingestão de alimento motivada pelo efeito do clima traz consequências diretas no tempo de alimentação, ruminação e ócio (Indu et al., 2015).

A elevação na ingestão de água acaba sendo uma das principais respostas que o animal apresenta com o intuito de se manter em condições de homeostasia. Os requerimentos de água variam e são regulados por muitos fatores, tais como consumo de matéria seca, temperatura ambiente e perda de água dos tecidos do corpo (Marai et al. 2007), bem como pelo tipo de alimento consumido, e pela idade e o estado fisiológico do animal (Araujo et al., 2011).

O consumo médio de água de um ovino é de 3 a 4 litros por dia, podendo chegar até 10 litros (Hafez, 1973). Dixon et al. (1999) verificaram consumo de 5,62 e 2,46 L/d, para borregos mantidos em temperatura de 32 a $40^{\circ} \mathrm{C}$ e de 13 a $15^{\circ} \mathrm{C}$, respectivamente $(\mathrm{P}<0,01)$.

\section{Índices do ambiente térmico}

A determinação do impacto do ambiente climático nas respostas dos animais de criação normalmente é feita através da combinação das diferentes variáveis climáticas em um índice, que expressa o conforto térmico do animal (Barbosa e Silva, 1995; Hahn et al., 2009).

Para a espécie ovina, Barbosa e Silva (1995) desenvolveram o ICT (Índice de Conforto Térmico), o qual relaciona as variáveis: temperatura do ar, pressão parcial de vapor, temperatura de globo negro e velocidade do vento, e, mais recentemente, Marai et al. (2007), geraram o ITU ovino. Este, foi testado na Grécia, de 2007 a 2012, por Papanastasiou et al. (2015), quando avaliaram as condições de estresse térmico em ovinos durante o verão, assim classificando-os de acordo com as condições meteorológicas prevalentes. A avaliação mostrou que o valor horário máximo diário excedeu ao limiar de estresse térmico em $82 \%$ dos dias, com ITU maior 
que 25,6, considerado estresse extremamente severo.

\section{O estresse térmico e o desempenho produtivo}

A meta na produção econômica de ovinos de corte é a capacidade de transformar o maior volume possível de nutrientes consumidos em músculo, estando o consumo de alimentos e o ganho de peso diretamente relacionados à eficiência alimentar dos animais.

A capacidade de consumo dos animais oscila em função do peso vivo e da taxa de ganho de peso, sendo ainda determinada pela genética, nutrição, sanidade, instalações e pelo clima (Cabral et al., 2008). O consumo de alimentos pelos ruminantes, segundo os mesmos autores, é controlado por mecanismos físicos, fisiológicos ou psicogênicos. Quando os animais são mantidos a base de dietas com elevado teor de concentrado, é possível que o principal mecanismo envolvido na regulação do consumo seja o fisiológico, assim as necessidades em nutrientes (energia) dos animais acabam regulando o consumo.

Quando os mecanismos fisiológicos do animal falham por excesso de carga térmica, a temperatura retal aumenta e o animal sofre as consequências do estresse. $\mathrm{O}$ estresse térmico evoca uma série de mudanças drásticas nas funções biológicas dos animais, as quais incluem diminuição do consumo, distúrbios no metabolismo da água, proteínas, energia, balanço de minerais, reações enzimáticas, secreção hormonal e metabólitos sanguíneos (Rodrigues et al., 2010). Este conjunto de efeitos negativos sobre as funções biológicas resulta em prejuízos da produção, reprodução e sanidade (Marai et al., 2007; Kumar et al., 2011). A diminuição no consumo de alimentos ocorre nos animais como forma de reduzir a carga térmica. Marai et al. (2007) enfatizam serem os efeitos deletérios agravados quando a alimentação inclui alta porcentagem de forragem. Assim, em condições de forte estresse térmico a alimentação de ovelhas pode ser realizada com baixo teor de forragem durante o período mais fresco do dia. A concentração de fibra na dieta de ovinos tem relação direta com o consumo de matéria seca quando os animais se encontram em estresse térmico.

Ao trabalharem com borregos da raça Awasi mantidos em câmaras climatizadas, na temperatura de $32^{\circ} \mathrm{C}$, Bhattacharya e Hussan (1974) verificaram que o consumo de matéria seca diminuiu $14 \%$ quando os ovinos receberam dieta com $25 \%$ de forragem (feno de cevada) e em $50 \%$ quando a porcentagem de forragem na dieta foi de $75 \%$. Os mesmos autores observaram ainda que ao aumentar o percentual de forragem na dieta, a digestibilidade da proteína bruta e do extrato etéreo foi reduzida $(\mathrm{P}<0,05)$, embora não tenham sido observados efeitos na digestibilidade da matéria seca e da fibra bruta.

É consenso entre os estudiosos que altas temperaturas são determinantes nas diminuições no consumo de matéria seca e, consequentemente, diminuição do ganho de peso (Dixon et al., 1999; Bernabucci et al., 2009; Indu et al., 2015). Dixon et al. (1999) observaram aumento significativo $(\mathrm{P}<0,05)$ na digestibilidade da matéria seca consumida por cordeiros mantidos no calor, resultado da menor velocidade de passagem da ingesta pelo trato gastrointestinal, causado pelo menor consumo de alimento (Church, 1974). No entanto, esta constatação na literatura é contraditória. É reconhecido que o estresse calórico afeta negativamente a funcionalidade do rumem, seja por alteração do $\mathrm{pH}$, pelo equilíbrio ácidobase, pela alteração da flora ruminal, pela menor motilidade, menor produção de saliva (Kadzere et al., 2002) e pela diluição do conteúdo ruminal devido a maior ingestão de água (Bernabucci et al., 1999).

A redução no consumo de matéria seca, especialmente de fibra, em condições ambientais termicamente desfavoráveis, é uma tentativa do animal de adequar fisiologicamente o balanço entre produção e perda de calor. Com a redução no consumo de matéria seca, diminui a taxa metabólica e consequente a produção de calor (Mendes et al., 2014). A ingestão de matéria seca nem sempre pode declinar, especialmente quando as temperaturas noturnas são baixas, permitindo a recuperação da temperatura do dia, ou se a exposição às condições de calor for de curta duração (Gaughan et al., 2002). As alterações nos consumos de matéria seca são afetadas também pela densidade energética da dieta, condições do animal, exposição prévia as condições de calor e dias de alimentação (Gaughan et al., 2002). As respostas animais, em geral, variam de acordo com o tempo de mudança térmica; quando a mesma for de curto período, os animais apresentam mudanças comportamentais, fisiológicas e imunológicas (Nienaber e Hahn, 2007); quando ocorrerem por períodos prolongados, causam resposta no desempenho, isto é, no consumo e na perda de calor com reflexos no crescimento, reprodução e eficiência (Bernabucci et al., 2009). 
O conhecimento científico que hoje se dispõe sobre nutrição animal, em especial de animais ruminantes é de que quando o animal se encontra em condições de estresse calórico seu metabolismo é alterado (Marai et al., 2007), precisando então de ajustes nutricionais. Dixon et al. (1999) mostram haver evidências que as interações entre dieta e estresse térmico podem alterar as necessidades de energia metabolizável e de aminoácidos absorvidos, mudando os requerimentos de energia e proteína em ambientes tropicais. Além do manejo nutricional, possíveis modificações do meio ambiente de criação, e de uso de animais com maior grau de adaptação são práticas possíveis para diminuir custos na produção (Silva et al., 2015).

A simples seleção de animais com alta capacidade produtiva também é prática questionável, sendo necessário considerar a produtividade obtida de acordo com o consumo. A identificação e seleção de animais que usam o alimento mais eficientemente, sem comprometer a produção e as características de qualidade da carne, são importantes considerando o impacto econômico e ambiental da alimentação nos sistemas de produção (Fidelis et al., 2017). Para tanto, diversos índices desenvolvidos para avaliar a eficiência biológica devem embasar a seleção, tais como a conversão e eficiência alimentar, o consumo alimentar residual, a relação de Kleiber e o índice nutricional multivariado biológico (Gomes, 2015; Fidelis et al., 2017).

O consumo alimentar residual tem sido uma ferramenta muito usada em melhoramento animal (Gomes, 2015), sendo aceito como uma medida de eficiência alimentar que independe da taxa de crescimento e do tamanho corporal adulto do animal. Fazendo isso, evitam-se comparações errôneas entre animais de diferentes portes e o favorecimento de animais de maior tamanho corporal. Animais de baixo consumo alimentar residual são mais eficientes pelo seu maior aproveitamento dos nutrientes da dieta (Magnani et al., 2013).

\section{Considerações Finais}

Os fatores climáticos acarretam respostas fisiológicas e comportamentais marcantes nos ovinos, e, quando essas condições atingem graus elevados de estresse, acabam impactando fortemente nos índices de produtividade.

É necessário adequar práticas de manejo, instalações e alimentação de tal forma que permitam melhores condições de bem-estar dos animais frente a condições climáticas estressantes, bem como selecionar biótipos mais adaptados as características climáticas nas quais serão explorados, buscando permitir que expressem todo seu potencial produtivo.

\section{Referências}

Alhidary, I.A.; Shini, S.; Al Jassin, R.A.M.; Gaughan, J.B. Physiological responses of Australian Merino wethers exposed to high heat load. Journal of Animal Science, 90(1): 212-220, 2013.

Araujo, G.G.L.; Voltolini, T.V.; Turco, S.H.N.; Pereira, L.G.R. Água nos sistemas de produção de caprinos e ovinos. In: Voltolini, T.V. (ed.) Produção de ovinos e caprinos no Semiárido. Petrolina: Embrapa, 2011. p.6994.

Baêta, F.C.; Souza, C.F. Ambiência em edificações rurais. $2^{\mathrm{a}}$ ed. Viçosa: UFV, 2012. $269 p$.

Barbosa, O.R.; Silva, R.G. Índice de conforto térmico para ovinos. Boletim de Indústria Animal, 52(1): 29-35, 1995.

Baumgard, L.H.; Rhoads, R.P. Ruminant nutrition symposium: Ruminant production and metabolic responses to heat stress. Journal of Animal Science, 90(6): 1855-1965, 2012.

Bernabucci, U.; Bani, P.; Ronchi, B.; Lacetera, N.; Nardone, A. Influence of short and long-term exposure to hot environment on rumen passage rate and diet digestibility by Frisian heifers. Journal of Dairy Science, 82(5): 967-973, 1999.

Bernabucci, U.; Lacetera, N.; Danieli, P.P.; Bani, P.; Nordane, A.; Ronchi, B. Influence of different periods of exposure to hot environment on rumen function and diet digestibility in sheep. International Journal of Biometeorology, 53(5): 387-395, 2009.

Blanes, V.V.; Guijarro, E.; Balasch, S.; Torres, A.G. Application of computational fluid dynamics to the prediction of airflow in a mechanically ventilated commercial poultry building. Biosystems Engineering, 100(1): 105-116, 2008.

Bhattacharya, A.N.; Hussain, F. Intake and utilization of nutrients in sheep fed different levels of roughage under heat stress. Journal of Animal Science, 38(4): 877-886, 1974.

Bridi, A.M. Efeitos do ambiente tropical sobre a produção animal. 2010a. 18p. Disponível em: 
<www.uel.br/pessoal/ambridi/Bioclimatologi a_arquivos/EfeitosdoAmbienteTropicalsobre aProducaoAnimal.pdf>. Acesso em: 30 mar. 2017.

Bridi, A.M. Adaptação e aclimatação animal. 2010b. 15p. Disponível em: <www.uel.br/pessoal/ambridi/Bioclimatologi a_arquivos/AdaptacaoeAclimatacaoAnimal.p df>. Acesso em: 29 fev. 2017.

Broom, D.M.; Molento, C.F.M. Bem-estar animal: conceito e questões relacionadas - Revisão. Archives of Veterinary Science, 9(2): 01-11, 2004.

Cabral, L.S.; Neves, E.M.O.; Zervoudakis, J.T.; Abreu, J.G.; Rodrigues, R.C.; Souza, A.L.; Oliveira, I.S. Estimativas dos requisitos nutricionais de ovinos em condições brasileiras. Revista Brasileira de Saúde e Produção Animal, 9(3): 529-542, 2008.

Costa, M.J.R.P. Ambiência na produção de bovinos de corte. Anais de Etologia, 18(1): 26-42, 2000.

Chulayo, A.Y.; Muchenje, V. A balanced perspective on animal welfare for improved meat and meat products: a review. South African Journal Animal Science, 45(5): 0127, 2015.

Church, D.C. Fisiologia digestiva y nutrición de los ruminantes. Zaragoza: Acribia, 1974. 483p.

Degen, A.A.; Shkolnik, A. Thermoregulation in fat-tailed Awassi, a desert sheep, and in german mutton Merino, a mesic sheep. Physiological Zoology, 51(4): 333-339, 1978.

Dixon, R.M.; Thomas, R.; Holmes, J.H.G. Interations between heat stress and nutrition in sheep fed roughage diets. Journal of Agricultural Science, 132(3): 351-359, 1999.

Eckert, R. Fisiologia animal: mecanismos e adaptações. $4^{\mathrm{a}}$ ed. São Paulo: Guanabara, 2000. 729p.

Eloy, Â.M.X. Estresse na produção animal (Comunicado Técnico). Brasília: Embrapa, 2007. 7p.

Encarnação, R.O. Estresse e produção animal. Campo Grande: Embrapa 2a ed., 1992. 33p. Disponível em: https://docsagencia.cnptia.embrapa.br/bovinode corte/doc/doc34/doc34.pdf. Acesso em: 05 mai. 2017.

Fidelis, H.A.; Bonilha, S.F.M.; Tedeschi, L.O.; Branco, R.H.; Cyrillo, J.N.S.G.; Mercadante, M.E.Z. Residual feed intake, carcass traits and meat quality in Nelore cattle. Meat Science, 128(1): 34-39, 2017.
Gaughan, J.B.; Mader, T.L.; Holt, S.M.; Hahn, G.L.; Young, B.A. Review of current assessment of cattle and microclimate during periods of high heat load. Australian Society of Animal Production, 24(1): 77-80, 2002.

Gomes, R.C. Entendendo a eficiência alimentar. Campo Grande: Embrapa, 2015. 3p. Disponível em: < https://ainfo.cnptia.embrapa.br/digital/bitstrea $\mathrm{m} /$ item/131582/1/Sumario-Senepol-2015-2paginas-22-23-e-24.pdf>. Acesso em: 22 jun. 2017.

Hafez, E.S.E. Adaptación de los animales domésticos. Barcelona: Labor, 1973. 563p.

Hahn, G.L.R.; Gaughan, J.B.; Mader, T.L.; Eigenberg, R.A. Thermal indices and their applications for livestock environments. In: De Shazer, J.A. (ed.) Livestock Energetics and Thermal Environmental Management. $1^{\mathrm{a}}$ ed. Mich: ASABE, 2009. p.113-130.

Indu, S.; Sejian, V.; Kumar, D.; Pareek, A.; Naqvi, S.M.K. Ideal proportion of roughage and concentrate for Malpura ewes to adapt and reproduce in a semi-arid tropical environment. Tropical Animal Health Production, 47(8): 1487-1495, 2015.

Kadzere, C.T.; Murphy, M.R.; Silanikove, N.; Maltz, E. Heat stress in lactating dairy cows: a review. Livestock Production Science, 77: 59-91, 2002.

Kuczinski, T.; Vidal, V.B.; Li, B.; Gates, R.S.; Nääs, I.A.; Moura, D.J.; Berckmans, D.; Banhazi, T.M. Impact of global climate change on the health, welfare and productivity of intensively housed livestock. International Journal of Agricultural and Biological Engineering, 4(2): 01-22, 2011.

Kumar, B.V.S.; Ajeet, K; Meena, K. Effect of heat stress in tropical livestock and different strategies for its amelioration. Journal of Stress Physiology \& Biochemistry, 7(1): 4554, 2011.

Magnani, E.; Nascimento, C.F.; Branco, R.H.; Bonilha, S.F.M.; Ribeiro, E.G.; Mercadante, M.E.Z. Relações entre consumo alimentar residual, comportamento ingestivo e digestibilidade em novilhas Nelore. Boletim de Indústria Animal, 70(2): 187-194, 2013.

Marai, I.F.M.; Darawany, A.A.; Fadiel, A.; Hafez, M.A.M.A. Physiological traits as affected by heat stress. Small Ruminant Research, 71(13): 01-12, 2007. 
Mendes, A.M.P.; Azevedo, M.; Lopes, P.M.O.; Moura, G.B.A. Zoneamento bioclimático para a raça Dorper no Estado de Pernambuco. Pesquisa Agropecuária Brasileira, 49(12): 986-993, 2014.

Moyes, C.D.; Schulte, P.M. Fisiologia térmica. In: Princípios de fisiologia animal, $2^{\mathrm{a}}$ ed. Porto Alegre: Artmed, 2010. 792p.

Neiva, J.N.M.; Teixeira, M.; Turco, S.H.N.; Oliveira, S.M.P.; Moura, A.A.A.N. Efeito do estresse climático sobre os parâmetros produtivos e fisiológicos de ovinos Santa Inês em confinamento na região litorânea do Nordeste do Brasil. Revista Brasileira de Zootecnia, 33(3): 668-678, 2004.

Nienaber, J.A.; Hahn, G.L. Livestock production system management responses to thermal challengs. International Journal of Biometeorology, 52(2): 149-157, 2007.

Oliveira, C.B.; De Bortolli, E.C.; Barcellos, J.O.J. Diferenciação por qualidade da carne bovina: a ótica do bem-estar animal. Ciência Rural, 38(7): 2092-2096, 2008.

Paim, T.D.P.; Borges, B.O.; Lima, P.M.T.; Dallago, B.; Louvandini, H.; McManus, C. Relation between thermographic temperatures of lambs and thermal comfort indices. International Journal of Applied Animal Sciences, 2, 1(4), 108-115, 2012.

Papanastasiou, D.K.; Bartzanas, T.; Kittas, C. Classification of potential sheep heat-stress levels according to the prevailing meteorological conditions. Agricultural Engineering International: CIGR Journal, Special issue: 57-64, 2015.

Polli, V.A.; Vaz, R.Z.; Carvalho, S.; Costa, P.T.; Mello, R.O.; Restle, J.; Nigeliskiic, A.F.; Silveira, I.D.B.; Pissinin, D. Thermal comfort and performance of feedlot lambs finished in two climatic conditions. Small Ruminant Research, 174: 163-169, 2019.

Randall, M. The physiology of stress: cortisol e hypothalamic-pituitary-adrenal axis. Dartmouth Undergraduate Journal Science, 3(1): 22-24, 2010.

Roberto, J.V.B.; Souza, B.B. Utilização da termografia de infravermelho na medicina e na produção animal. Journal Animal
Behavior Biometeorology, 2(3): 73-84, 2014.

Rodrigues, N.E.B.; Zangeronimo, M.G.; Fialho, E.T. Adaptações fisiológicas de suínos sob estresse térmico. Revista Eletrônica Nutritime, 7(2): 1197-1211, 2010.

Schmidt-Nielsen, K. Fisiologia animal. São Paulo: Santos, $5^{\mathrm{a}}$ ed., 2005. 611p.

Silanikove, N. Effects of heat stress on the welfare of extensively managed domestic ruminants. Livestock Production Science, 67(1-2): 01$18,2000$.

Silva, G.A; Souza, B.B.; Silva, E.M.N. Adaptabilidade de ovinos e estratégias para amenizar os efeitos do clima em regiões tropicais. Journal of Animal Behaviour and Biometeorology, 3(1): 20-27, 2015.

Silva, M.R.; Souza, B.B.; Guimarães, L.J.; Costa, D.F.; Rocha, E.D.; Souto, D.V.O.; Silva, E.M.N. Estresse térmico e sua influência na fisiologia hormonal de pequenos ruminantes. Journal of Animal Behaviour and Biometeorology, 4(2): 50-54, 2016.

Silva, R.G. Introdução à bioclimatologia animal. São Paulo: Nobel, 2000. 286p.

Souza, B.B.; Batista, N.L. Os efeitos do estresse térmico sobre a fisiologia animal. Agropecuária Científica no Semiárido ACSA, 8(3): 06-10, 2012.

Srikandakumar, A.; Johnson, E.H.; Mahgoub, O. Effect of heat stress on respiratory rate, rectal temperature and blood chemistry em Omami and Australian Merino sheep. Small Ruminant Research, 49(2): 193-198, 2003.

St. Pierre, N.R.; Cobanou, B.; Schnitkey, G. Economic losses from heat stress by US livestock industries. Journal of Dairy Science, 86(Suppl.): E52-E77, 2003.

Starling, J.M.C.; Silva, R.G.; Muñoz, M.C.; Barbosa, G.S.S.C.; Costa, M.J.R.P. Análise de algumas variáveis fisiológicas do grau de adaptação de ovinos submetidos ao estresse por calor. Revista Brasileira de Zootecnia, 31(5): 2070-2077, 2002.

Wojtas, K.; Cwynar, P.; Kolacz, R.; Kupczynski, R. Effect of heat stress on acid-base balance in Polish Merino sheep. Archiv fur Tierzucht, 56(92): 917-923, 2013. 